\title{
The Unnecessity of Positron Emission Tomography Computed Tomography in the Etiologic Evaluation of Neurodevelopmental Delay in Craniosynostosis Patients
}

\author{
Chae Eun Yang ${ }^{1^{\star}}$, \\ Eun Kyung Park ${ }^{2^{*}}$, \\ Myung Chul Lee ${ }^{3}$, \\ Kyu Won Shim ${ }^{2^{\star *}}$, \\ Yong Oock Kim ${ }^{1 \star \star}$ \\ ${ }^{1}$ Institute for Human Tissue Restoration, \\ Department of Plastic and Reconstructive \\ Surgery, Severance Hospital, Yonsei University \\ College of Medicine, Seoul; \\ ${ }^{2}$ Department of Pediatric Neurosurgery, \\ Severance Children's Hospital, Yonsei \\ University College of Medicine, Seoul; \\ ${ }^{3}$ Department of Plastic and Reconstructive \\ Surgery, Konkuk University School of \\ Medicine, Seoul, Korea \\ No potential conflict of interest relevant to \\ this article was reported.
}

\begin{abstract}
Background: In evaluation of craniosynostosis patients in terms of neurodevelopmental delay, positron emission tomography computed tomography (PET-CT) scan can be used to assess brain abnormalities through glucose metabolism. We aimed to determine the unnecessity of PET-CT in this study.

Methods: Thirty-eight patients diagnosed with craniosynostosis who underwent distraction osteogenesis from October, 2010 to November, 2013 were reviewed. Magnetic resonance imaging (MRI) and PET-CT scan were carried out for evaluation of the brain structure and function, whereas X-ray and CT scan were taken for evaluation of the skull. Results: Nine patients reported abnormal MRI findings which were not significant, and five patients showed local problem on brain on PET-CT scan. No correlation was found among them.

Conclusion: PET-CT evaluation of possible abnormal brain findings do not affect surgical planning or require additional therapy. Preoperative PET-CT scan is not the essential study to get any etiologic information of the disease consequences or to establish the treatment plan.
\end{abstract}

Keywords: Craniosynostosis / Positron emission tomography computed tomography / Evaluation

\section{INTRODUCTION}

Craniosynostosis is the premature closure of one or more cranial vault sutures, usually resulting in an abnormal cranial shape. Restricted growth and compensatory bossing can be associated with increased intracranial pressure and can lead to delayed neurodevelopment.

Inspection and palpation are critical for the diagnosis of cra-

\footnotetext{
Correspondence: Yong Oock Kim

Institute for Human Tissue Restoration, Department of Plastic and Reconstructive Surgery, Severance Hospital, Yonsei University College of Medicine, 50-1 Yonsei-ro, Seodaemungu, Seoul 03722, Korea

E-mail: SGM625@yuhs.ac

Kyu Won Shim

Department of Pediatric Neurosurgery, Severance Children's Hospital, Yonsei University College of Medicine, 50-1 Yonsei-ro, Seodamungu, Seoul 03722, Korea

E-mail: shimkyuwon@yuhs.ac

*The first two authors contributed equally to this work. ${ }^{* *}$ These authors contribited equally.
}

Received December 20, 2016 / Revised February 6, 2017 / Accepted February 7, 2017 niosynostosis. Cephalic index and head circumference are supportive means of diagnosis. To differentiate these patients from positional plagiocephaly, three-dimensional computed tomography (3D-CT) scan is necessary. It is valuable to document the suture involved and determine extent of synostosis. Magnetic resonance imaging (MRI) is also used to determine intracranial anomalies and sometimes substitute CT scan to avoid ionizing radiation.

Positron emission tomography computed tomography (PETCT) can be tried to assess the perfusion state of brain or brain malformations. Some authors reported that abnormal cerebral perfusion is found among craniosynostosis patients which can bring functional consequences [1-3]. David et al. [3] reported that surgical release and cranial reconstructive surgery may improve perfusion abnormalities. But PET-CT requires fasting, sedation and intravenous injection to the patients and the additional eco- 
nomic burden is needed.

In this study, we aimed to find the relationship between abnormal cerebral perfusion and craniosynostosis and determine whether this study is necessary to evaluate the disease consequences and establish the treatment plan.

\section{METHODS}

This study was approved by the Yonsei University Institutional Review Board and all forms of data was collected after obtaining informed consent from guardians. Thirty-eight patients diagnosed with craniosynostosis who underwent distraction osteogenesis by a craniofacial surgeon as well as pediatric neurosurgeons from October, 2010 to November, 2013 were reviewed. Suspicious patients with abnormal physical findings of their skull shape had taken plain skull X-ray and were recommended to carry out a three dimensional computed tomography (3D-CT). The diagnosis was established after confirmation of premature closure of more than one suture on the 3D-CT images. Preoperative brain magnetic resonance imaging (MRI) was performed to evaluate any combined structural brain abnormality. It was carried out with a 1.5-tesla magnetic resonance unit (Intera Achieva, Philips Medical Systems, Best, The Netherlands) in axial, coronal, and sagittal planes. To evaluate any functional abnormality of brain through brain metabolism, PET-CT scans were taken by a combined PET-CT system (Discovery LS, GE Healthcare, Milwaukee, WI, USA) which integrates a PET scanner (DSTe, GE Healthcare) with a multislice helical CT (16 slice, GE Healthcare) which permits the acquisition of co-registered CT and PET images in the same session. Before scanning, patients fasted for 6 hours and sedated during the procedure. Twenty minutes after a standard dose of $145 \mathrm{mCi} / \mathrm{kg}$ of F18-fluoro-2-deoxyglucose (FDG) was administered, imaging was performed using a diagnostic high-dose CT scan (scan field of $300 \mathrm{~mm}$, increment of $3 \mathrm{~mm}$, slice thickness 2.3 $\mathrm{mm}$, pitch of 1.5 -s per rotation, matrix $512 \times 512,120 \mathrm{KV}, 450$ $\mathrm{mAs}$ ). PET images were reconstructed by a standard two-dimensional iterative algorithm (ordered subset expectation maximization). Experienced pediatric neurologists as well as neuroradiologists analyzed the MRI imaging and nuclear radiologist evaluated the PET-CT imaging.

\section{RESULTS}

Total thirty-eight patients of sixteen girls and twenty two boys have diagnosed as nonsyndromic craniosynostosis and undergone distraction osteogenesis. Mean age at diagnosis was 20.8土 17.5 months, ranges from 4 months to 80 months (Table 1).

On preoperative imaging study, abnormal findings on MRI were detected in nine patients (23.7\%) such as Chiari malformation, whereas the other findings of twenty-nine patients were considered normal (76.3\%). Two patients who showed small volume on frontal lobe had craniosynostosis on the same side which thought to be a consequence of structural deformity of the skull. On PET-CT study, five patients (13.2\%) showed abnormal findings. In four patients (10.5\%), decreased FDG uptake was reported on certain lobes, and one patient showed cortical atrophy and fissure widening (Figs. 1, 2), while the other thirty-three patients (86.8\%) showed unremarkable PET-CT findings which showed normal metabolism of brain.

No correlation was found between the MRI and PET-CT findings. No patients with pathologic findings of the brain MRI showed abnormal FDG uptake of the brain on PET-CT, and vice versa (Table 2).

\section{DISCUSSION}

Nonsyndromic craniosynostosis is an isolated form of premature

Table 1. Patient demographic and clinical details

\begin{tabular}{lc} 
Characteristic & Value \\
Age $(\mathrm{mo})$ & $20.8 \pm 17.5(4-80)$ \\
Male & 22 \\
Female & 16 \\
Involved sutures & \\
Bicoronal & $5(13.2)$ \\
Unicoronal & $8(21.1)$ \\
Lamboidal & $8(21.1)$ \\
Metopic & $4(10.3)$ \\
Sagittal & $8(21.1)$ \\
Multiple & $5(13.2)$ \\
\hline
\end{tabular}

Values are presented as mean \pm standard deviation (ragne) or number (\%). 


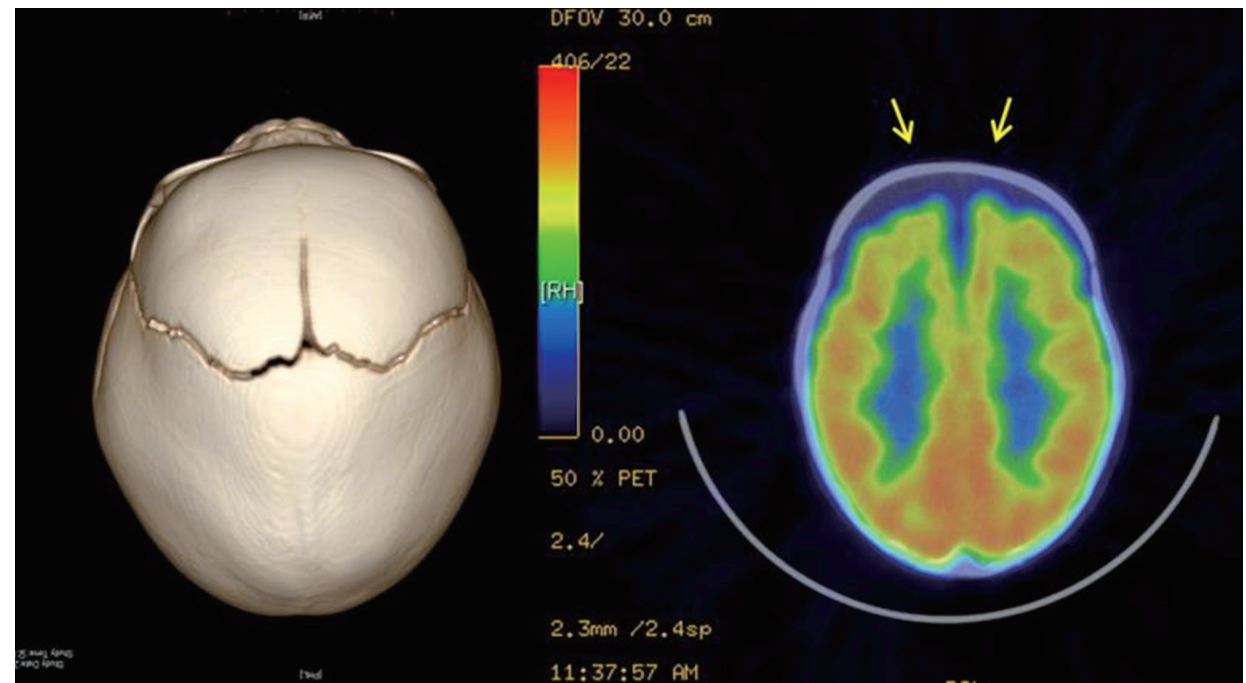

Fig. 1. Positron emission tomography computed tomography showing mild cortical atrophy in the frontal lobes and fissure widening (arrows) in 8 months-old girl who had sagittal craniosynostosis.

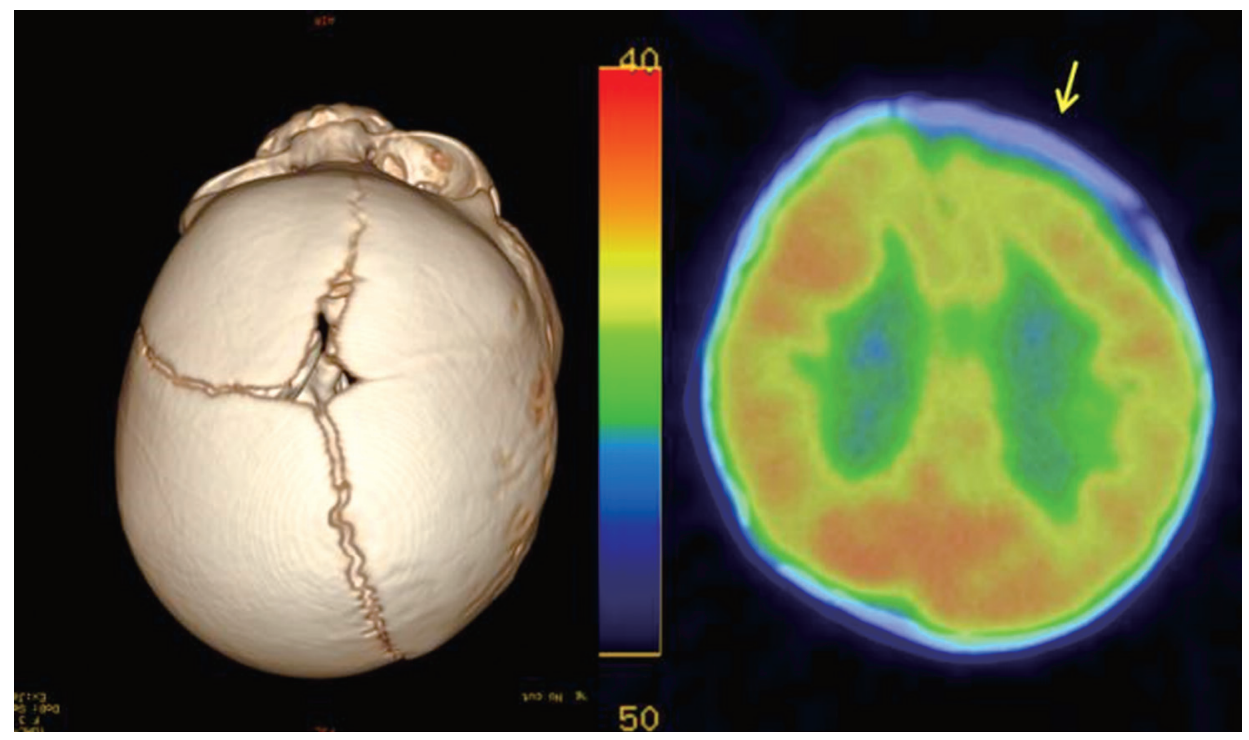

Fig. 2. Positron emission tomography computed tomography showing mild decreased F18-fluoro-2-deoxyglucose uptake in the left frontal lobe (arrow) in 4 months-old girl who had right coronal and right lambdoid craniosynostosis.

fusion of one or more cranial vault sutures without any associated genetic syndrome. It occurs in approximately 1 in 1,800 to 1 in 2,500 births, typically involving a single suture $[4,5]$. Risks of cognitive deficits, learning and language disabilities during infancy were reported with three-to-five higher tendency than normal groups [6,7]. It is still controversial whether these brain abnormalities are primary or secondary to the tension forces of skull defor- mation [8].

In this study, only $10.5 \%$ patients showed local hypoperfusion on preoperative PET-CT. This is much less compared to David et al.s [3] 1996 study that reported 71.4\% (five of seven patients) and 1999 study [9] that reported 80\% (eight of ten patients) of patients showed decreased blood flow. We concluded that preoperative PET-CT scan is not the essential study to get any etiologic infor- 
Table 2. Abnormal MRI and PET-CT study findings

\begin{tabular}{lll}
\hline Diagnosis & & \\
\hline Bicoronal & Small volume, frontal lobe, bilateral & No abnormal findings \\
\hline Unicoronal, Rt. & Small volume, frontal lobe, right & No abnormal findings \\
\hline Metopic & Chiari-malformation & No abnormal findings \\
\hline Sagittal & Dilated lateral ventricle & No abnormal findings \\
\hline Mixed (sagittal \& metopic) & R/O partial agenesis of corpus callosum & No abnormal findings \\
\hline Metopic & No abnormal findings & Decreased FDG uptake, frontal lobe, left \\
\hline Mixed (coronal \& lamdoid) & No abnormal findings & Decreased FDG uptake, frontal lobe, left \\
\hline Metopic & No abnormal findings & Decreased FDG uptake, frontal lobe, right \\
\hline Unicoronal, Lt. & No abnormal findings & Decreased FDG uptake, parietotemporal lobe, bilateral \\
Mixed (coronal \& lambdoid) & No abnormal findings & Mild cortical atrophy, frontal lobes, fissural widening \\
\hline
\end{tabular}

MRI, magnetic resonance imaging; PET-CT, positron emission tomography computed tomography; Rt., right; R/O, rule out; FDG, F18-fluoro-2-deoxyglucose; Lt., left.

mation of the disease progression to developmental delay or to establish the treatment plan. In terms of the cause for the tendency of developmental delay, chronically raised intracranial pressure and alteration in brain structure were suggested [10]. Neuropsychological functions develops as growing, so continuous craniosynostotic force would hamper normal development.

Skull distortion is suggested for the cause for the higher tendency of children with nonsyndromic craniosynostosis having neurodevelopmental impairment. Developmental delay become more prominent as the patients grow, which supports that it is not the matter of the brain itself. PET-CT scan is not necessarily needed for evaluation of nonsyndromic craniosynostotic patients for this reason. Evaluation of possible abnormal brain findings do not affect surgical planning or require additional therapy. Even though the cause of developmental impairment and the optimal type of surgical procedure are still controversial, it seems that early operation for correction of skull dysmorphology should be considered in nonsyndromic craniosynostosis patients. We can expect not only better appearance but also better long-term intellectual improvement.

\section{REFERENCES}

1. Sen A, Dougal P, Padhy AK, Bhattacharya A, Kumar R, Bal C, et al.
Technetium-99m-HMPAO SPECT cerebral blood flow study in children with craniosynostosis. J Nucl Med 1995;36:394-8.

2. Engel M, Hoffmann J, Muhling J, Castrillon-Oberndorfer G, Seeberger R, Freudlsperger C. Magnetic resonance imaging in isolated sagittal synostosis. J Craniofac Surg 2012;23:e366-9.

3. David LR, Wilson JA, Watson NE, Argenta LC. Cerebral perfusion defects secondary to simple craniosynostosis. J Craniofac Surg 1996;7:177-85.

4. Di Rocco F, Arnaud E, Renier D. Evolution in the frequency of nonsyndromic craniosynostosis. J Neurosurg Pediatr 2009;4:21-5.

5. Selber J, Reid RR, Chike-Obi CJ, Sutton LN, Zackai EH, McDonaldMcGinn D, et al. The changing epidemiologic spectrum of single-suture synostoses. Plast Reconstr Surg 2008;122:527-33.

6. Kapp-Simon KA, Speltz ML, Cunningham ML, Patel PK, Tomita T. Neurodevelopment of children with single suture craniosynostosis: a review. Childs Nerv Syst 2007;23:269-81.

7. Speltz ML, Kapp-Simon KA, Cunningham M, Marsh J, Dawson G. Single-suture craniosynostosis: a review of neurobehavioral research and theory. J Pediatr Psychol 2004;29:651-68.

8. Raybaud C, Di Rocco C. Brain malformation in syndromic craniosynostoses, a primary disorder of white matter: a review. Childs Nerv Syst 2007;23:1379-88.

9. David LR, Genecov DG, Camastra AA, Wilson JA, Argenta LC. Positron emission tomography studies confirm the need for early surgical intervention in patients with single-suture craniosynostosis. J Craniofac Surg 1999;10:38-42.

10. Starr JR, Lin HJ, Ruiz-Correa S, Cunningham ML, Ellenbogen RG, Collett BR, et al. Little evidence of association between severity of trigonocephaly and cognitive development in infants with single-suture metopic synostosis. Neurosurgery 2010;67:408-15. 